\title{
Informationsaustausch in Prototypingprozessen: Bestimmung und Beschreibung von Störgrößen
}

\author{
Information Exchange in Prototyping Processes: Determination and Description of \\ Disturbance Variables
}

\author{
Simon J. Nicklas ${ }^{1 *}$, Kristin Paetzold ${ }^{1}$ \\ ${ }^{1}$ Institute for Technical Product Development, University of the Bundeswehr Munich \\ ${ }^{*}$ Korrespondierender Autor: \\ Simon J. Nicklas \\ Universität der Bundeswehr München \\ Werner-Heisenberg-Weg 39 \\ 85579 Neubiberg \\ Telefon: +4989 6004-2391 \\ Mail: simon.nicklas@unibw.de
}

\begin{abstract}
Innovation requires methodologies that enable the continuous development of effective and context-relevant solutions. Thus, the frequent comparison of development goals and actual user needs is of crucial importance. Prototyping is a widely used tool for User Integration that supports the mutual process of understanding between designer and user. Yet, this usage of nonverbal communication in the course of the process is ambiguous and brings along its specific challenges for the designer. An enhanced system description of the prototyping process seems necessary to provide the developer with wellfounded and comprehensible guidelines. Therefore, the present work analyses the similarities and differences of disturbance variables the designer may encounter in different prototyping processes.
\end{abstract}

\author{
Keywords \\ prototyping, user integration, communication gap, design theory, \\ cybernetics
}




\section{Einleitung}

Innovation, als zentrales Mittel zur Wettbewerbsfähigkeit eines Unternehmens, erfordert Methodiken, die die kontinuierliche Entwicklung von wirkungsvollen und kontext-relevanten Lösungen ermöglichen. Gerade im Kontext von agilen und sogenannten hybriden Vorgehensmodellen ist der regelmäßige Abgleich von Entwicklungsziel und tatsächlichem Nutzerbedarf von entscheidender Bedeutung. In diesem Kontext haben Methoden der Nutzerintegration in den letzten Jahren stark an Bedeutung gewonnen, um die Produktentwicklung stärker an den Nutzerbedarfen zu orientieren [1]. Auch im Zuge der zunehmenden Verbreitung agiler Entwicklungsansätze ist die Bedeutung des Abgleichs von Entwicklungszielen mit dem Entwicklungskontext, respektive dem Nutzen der Produkte für den Kunden ${ }^{1}$, stärker in den Fokus getreten [2]. Ziel ist es dabei vor allem, tatsächliche Nutzerbedarfe zu identifizieren, um nicht nur das Produkt richtig zu entwickeln, sondern auch das richtige Produkt zu entwickeln [3].

Ein entscheidender Faktor für die erfolgreiche Integration des Nutzers in den Entwicklungsprozess ist die Fähigkeit, die Bedürfnisse der Nutzer durch Kommunikation ${ }^{2}$, also den Austausch von Information, zunächst zu ermitteln und im weiteren Verlauf auch entsprechend zu validieren (vgl. [5], [6]). Im Folgenden werden daher ausschließlich Formen der Nutzerintegration betrachtet, die eine entsprechende Kommunikation mit sich bringen. Typischerweise erleichtern Prototyping und Co-Design-Ansätze diesen Dialog, indem sie Lösungsansätze materialisieren und so dabei helfen, die zukünftige Nutzung eines Produkts in seinem vorgesehenen Kontext greif- und vorstellbar zu machen.

\section{Fragestellung}

Ein möglicher Ansatz zur Nutzerintegration und zur Überwindung der Kommunikationslücke zwischen Entwickler und Nutzer ist das Prototyping. Durch die Zuhilfenahme analoger, das heißt nonverbaler, Modalitäten - in diesem Fall also greif- und erlebbarer Artefakte - soll der gegenseitige Verständnisprozess hierbei gezielt unterstützt werden. Gerade diese analoge Kommunikation ist jedoch nicht eindeutig und bringt daher ganz eigene Herausforderungen für den Entwickler mit sich.

Da sozialwissenschaftlichen Kompetenzen in der ingenieurswissenschaftlichen Ausbildung eine zunehmend wichtige Bedeutung zukommt [7], scheint eine verbesserte Systembeschreibung des Prototyping-Prozesses samt vorhandener Störgrößen und Filter insofern notwendig, um dem Entwickler fundierte, aber auch verständliche Richtlinien an die Hand geben zu können, die es ihm ermöglichen, die auftretenden Einflussfaktoren im Prototypingprozess zu berücksichtigen. Um diesem Ziel näher zu kommen, werden in der vorliegenden Arbeit sowohl Gemeinsamkeiten als auch Unterschiede verschiedener Prototypingprozesse herausgearbeitet. Die entsprechende Forschungsfrage lässt sich analog wie folgt formulieren:

Welche kommunikativen Störgrößen treten im Prototypingprozess auf und wie müssen sie beschrieben werden, um sie im Prozessverlauf adressieren zu können?

\section{Forschungsansatz}

Im Rahmen der Untersuchung wurden zunächst verschiedene Definitionen des Prototypingbegriffs miteinander verglichen und Unterschiede wie Gemeinsamkeiten

\footnotetext{
${ }^{1}$ Zwischen Kunde und Nutzer bestehen signifikante Unterschiede, die den Autoren bewusst sind. Im Rahmen des Beitrags wird der Fokus auf den Nutzer gelegt. Alle folgenden Überlegungen lassen sich aber auch auf den Kunden übertragen.

${ }^{2}$ Für weitere begriffliche Definitionen auch außerhalb des Kontextes des vorliegenden Artikels sei auf die umfangreiche Aufarbeitung durch MERTEN [4] verwiesen.
} 
aufgezeigt. Auf Basis dieses konsolidierten Begriffsverständnisses werden bekannte Theorien zur Kommunikation, Kybernetik und Hermeneutik herangezogen, um im Rahmen eines deduktiven Ansatzes drei unterschiedliche Prototypingvarianten zu beschreiben, die sich hinsichtlich ihres üblichen Einsatzspektrums als auch ihrer jeweiligen Zielsetzung unterscheiden. Diese drei Konzepte werden mit Hilfe der zuvor eingeführten Begriffe verglichen und entsprechende Abgrenzungen gezogen, um mögliche, störende Einflussfaktoren identifizieren zu können. Die Ergebnisse werden zu Kommunikationsschemata zusammengefügt, die sowohl die Verbindungen als auch die Unterschiede aufzeigen, so dass entsprechende Konsequenzen für die Produktentwicklung in einem späteren Schritt abgeleitet werden können.

\section{Stand der Forschung}

\subsection{Kommunikation zur Integration des Nutzers}

Die Identifikation von Nutzerbedarfen ist nicht zuletzt deswegen eine Herausforderung, weil Entwickler und Nutzer Produkte, Ideen und Funktionalitäten unterschiedlich interpretieren. Während der Entwickler über den Systemzweck die Funktionen ableitet und konkretisiert, erschließt sich der Nutzer die Funktion des Produktes aus der konkreten Anwendung im Alltagsumfeld in seiner spezifischen Lebens- und Handlungssituation [8]. Dies führt zu unterschiedlichen Bewertungen, respektive Bewertungskriterien, sowohl beim Nutzer als auch beim Entwickler. Verständnisschwierigkeiten zwischen Entwicklern und Nutzern, SARODNICK \& BRAU [9] nennen dies "wechselseitige Ignoranz", führen zu Unsicherheiten in der Bewertung von Produktideen in der frühen Phase der Entwicklung. Diese können erheblichen wirtschaftlichen Schaden verursachen, wenn an den tatsächlichen Bedarfen vorbei entwickelt wird.

Ziel muss es daher sein, die Kommunikation zwischen Nutzer und Entwickler zu unterstützen und Störungen in dieser zu klären bzw. zu vermeiden. Zur theoretischen Beschreibung von Kommunikationsmodellen finden sich in der Literatur eine Reihe von Ansätzen (z.B. in [10]), die aber im Kontext der Nutzer-Entwickler-Kommunikation interpretiert und angepasst werden müssen. Interessant erscheinen in diesem Zusammenhang daher das grundlegende Informationsmodell nach SHANNON \& WEAVER [11] und die Axiome nach WATZLAWICK ET AL. [12], über die die Informationsübertragung als ein Sender-EmpfängerSystem beschrieben wird, bei dem Störungen zu einer Veränderung der Interpretation von Information führen können. Dies soll hier als Basis dienen. Die unterschiedlichen Sichtweisen von Entwicklern und Nutzern auf ein technisches System bedürfen demnach eines Transformationsprozesses, um die jeweiligen Intentionen und Ideen der Beteiligten dem jeweils anderen transparent und anschaulich zu machen.

\subsection{Prototypingmodelle}

Ein möglicher Ansatz zur Nutzerintegration und zur Überwindung der Kommunikationslücke zwischen Entwickler und Nutzer ist das Prototyping. Durch die Zuhilfenahme so genannter analoger Modalitäten, sprich nonverbaler Ausdrucksmittel wie Prototypen, soll der gegenseitige Verständnisprozess hierbei gezielt unterstützt werden. Gerade diese analoge Kommunikation ist nach WATZLAWICK ET AL. [12] jedoch mehrdeutig (vgl. viertes Axiom) und bringt daher ganz eigene Herausforderungen für den Entwickler mit sich. Durch den täglichen Gebrauch im akademischen Bereich, in der Industrie und in den Medien hat der Begriff "Prototyp" verschiedene Konnotationen erhalten. Um der daraus resultierenden taxonomischen Unschärfe zu begegnen, wurden mehrere Neologismen eingeführt, um die Bedeutung neu zu definieren:

Ein so genannter Provotyp ist ein provokativer Prototyp [13], der im Entwicklungsprozess genutzt wird, um eine Reaktion zu provozieren. Das Ziel ist es, Menschen so anzuregen und 
einzubeziehen, dass sie sich mögliche, zukünftige Situationen vorstellen (können). Der Schwerpunkt dieses Ansatzes liegt nicht in der Definition eines Problems (das heißt, der Überwindung unangenehmer Situationen), sondern vielmehr in der Erkundung von neuen Möglichkeiten. Auf diese Weise können einerseits Techniken zur Verfügung gestellt werden, die eine Vielzahl von Möglichkeiten ausloten, und andererseits können Unternehmen und Teilnehmer so provoziert werden, dass sie die mit einer Situation verbundenen Phänomene auf eine andere Art und Weise erleben [14].

Mit Hilfe von Pretotypen können die anfängliche Marktattraktivität und der reale Nutzen eines potenziellen neuen Produkts getestet werden, indem mit möglichst geringem Zeit- und Geldaufwand die entsprechende Kernerfahrung simuliert wird [15].

Unter dem verwandten Begriff des Minimum Viable Products (MVP) wiederum wird eine Strategie verstanden, bei der ein neues Produkt oder eine neue Dienstleistung mit gerade genügend Eigenschaften geschaffen wird, um den ersten Kunden zufrieden zu stellen. Diese so genannten Early Adopters sind bestrebt, etwas Neues auszuprobieren, im Gegenzug geben sie ihr Feedback dazu ab [16]. Auf diese Weise können zum Beispiel Start-Ups den "Bedingungen extremer Unsicherheit" begegnen, mit denen sie konfrontiert sind (ebd.), und für die die Entwicklung eines vollständigen Produkts vor der Konzepterprobung auf dem Markt riskant wäre. So entsteht die Möglichkeit, aus Experimenten zu lernen, bei denen eine frühe Version eines Produkts bereits gegen relevante Metriken getestet wird [17]. DUC UND ABRAHAMSSON [18] identifizieren die folgenden Anwendungen von MVPs: Unterstützung validierten Lernens, Erleichterung des Produktdesigns, Überbrückung von Kommunikationslücken und Erleichterung kostengünstiger Produktentwicklungsaktivitäten. Zusammenfassend lässt sich sagen, dass der Schwerpunkt der MVP in der Prüfung der Marktrelevanz einer Produktidee liegt.

Pirtyp: Im Gegensatz zu traditionellen Prototypen führt SCHMIDT [19] den Begriff des "Pirtype" ein. Pirtypes betonen den experimentellen Charakter der Entwicklung und lockern den Fokus auf die Körperlichkeit des Artefakts. Sie zielen vielmehr darauf ab, "[...] die Wissensbasis zu erweitern" (ebd.). Im Vergleich zu funktionsfähigen Inkrementen müssen Pirtypen nicht produktiv sein. Vielmehr wird darauf abgezielt, ein Produktinkrement mit der geplanten Nutzerbasis zu testen. Dementsprechend "verkörpern sie die aktuelle Wissensbasis, um diese zu validieren" (ebd.). [...] und helfen also, das Konzept in Richtung des beabsichtigten Ziels auszurichten, indem das Team ermutigt wird, auch neue Ansätze auszuprobieren und den Schwerpunkt auf das Lernen zu verlagern, wobei auch nichtphysische Artefakte zugelassen werden. (ebd.)

Die vorgenannten Definitionen zeigen, dass es diverse Unterschiede, aber auch gewisse Ähnlichkeiten zwischen den Begriffsdefinitionen gibt. Der Interpretation von HOUDE \& HILL [20] und SCHMIDT [19] ist insofern zuzustimmen, als dass der Prototyp nicht an bestimmte Vorgaben gebunden ist, insbesondere nicht daran, dass seine physikalische Beschaffenheit eine zwingende Voraussetzung sei. Wenn wir daher im folgenden Text den Begriff "Prototyp" verwenden, beziehen wir uns auf die Verwendung im Sinne von "Pirtyp", der physisch, digital oder gar virtuell sein kann.

Den meisten Prototypingmethodiken ist nichtsdestotrotz gemein, dass eine Verständnisgrenze zwischen Entwickler und Nutzer verbleibt: Der Prototyp als Objekt fungiert als Träger bestimmter, bedeutungsgeladener Zeichen [21], die auf Nutzerseite zunächst interpretiert werden müssen. Bisherige Ansätze, diese Grenze zu überwinden, vertrauen jedoch größtenteils auf der Erfahrung des Entwicklers und seinem Verständnis über die vorliegende Entwicklungsaufgabe.

\section{Ergebnisse}

Um den zugrundeliegenden Prozess analysieren zu können, bedarf es zunächst Beschreibungsansätzen zur Informationstransformation im Prototypingprozess. So beschreibt 
bereits das Sender-Empfänger-Modell nach SHANNON \& WEAVER [11] den Einfluss von Störungen auf die Informationsübertragung: Durch fehlerhafte Signale, Interferenzen oder eine unterschiedliche Haltung gegenüber der übertragenen Information ergeben sich Abweichungen zwischen der gesendeten und der empfangenen Nachricht. Das SenderEmpfänger-Modell, das seinen Ursprung in der Nachrichtentechnik hat, zeichnet demnach das Verhalten einer offenen Steuerung nach. Dabei codiert der Entwickler seine Produktintention in einem Prototyp mit den inm zur Verfügung stehenden Zeichen. Der Nutzer als Empfänger des Prototyps muss die darin enthaltenen Zeichen nun wieder decodieren, um sich die zugrundeliegende Idee zu erschließen (vgl. Bild 1). Im vorliegenden Kontext kann daraus abgeleitet werden, dass unvollständige Prototypen, darin enthaltene neue oder abweichende Zeichen und unterschiedliche Nutzungssituationen das Verständnis des Nutzers für den Prototyp beeinflussen, da die darin enthaltene Information von ihm nicht mehr vollständig oder nur fehlerhaft decodiert werden kann.

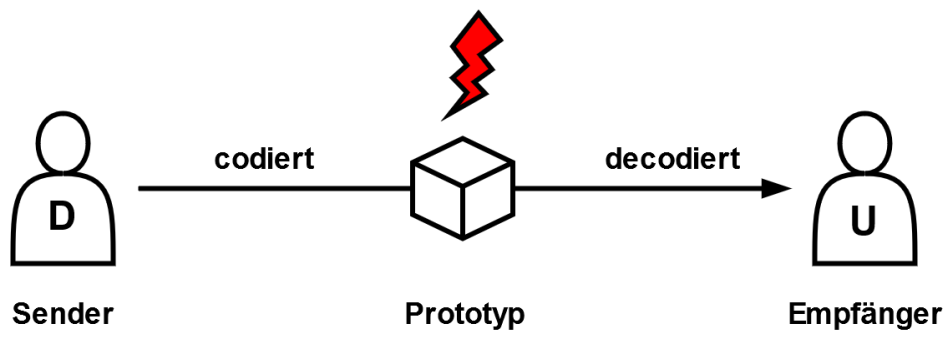

Bild 1: Informationsübertragung zwischen Entwickler (D) und Nutzer (U) in Anlehnung an SHANNON \& WEAVER [11]

Gemäß ROPOHL [22] kann das so entstandene sozio-technische System geschlossen und in einen kybernetischen Handlungskreis überführt werden. Dies deckt sich mit dem dritten Axiom nach WATZLAWICK ET AL. [12], das die Kommunikationsabläufe ebenfalls als Reaktionskette ohne definierten Anfang oder definiertes Ende beschreibt. Dabei dient im vorliegenden Fall das Feedback und Verhalten des Nutzers entsprechend als Rückführung der Regelgröße, so dass der Entwickler seine ursprüngliche Intention durch Kommunikation und Beobachtung gegen die tatsächliche Wahrnehmung und Erfahrung des Nutzers prüfen kann. Somit kann der Entwickler die Abweichung erkennen und versuchen, diese in darauffolgenden Iterationen zu minimieren. Die Störgrößen aus dem zuerst genannten Sender-Empfänger-Modell werden durch das Schließen des Systems zum Regelkreis jedoch nicht eliminiert, sodass beide Modelle zusammengeführt werden müssen. In diesem konsolidierten Modell können gemäß Bild 2 zwei Arten von Störgrößen identifiziert werden:

1. Der Umfang und die Darstellungsmethode des Prototyps entsprechen dem Zeichenvorrat des Shannon-Weaver-Modells. Fehlen die entsprechenden Anzeichenfunktionen ${ }^{3}$ des Prototyps oder verwendet der Prototyp mehr Zeichen als der Nutzer interpretieren kann, kann der Nutzer dessen intendierte Funktion nicht erschließen.

2. Das Verstehen des vorliegenden Prototyps durch den Nutzer in seinem eigenen Nutzungskontext hängt von der notwendigen Übereinstimmung der Zeichen im Shannon-Weaver-Modell ab: Unterschiedliche Erfahrungen führen zu Abweichungen in der Interpretation vorhandener oder neuer Zeichen - der Sinn des Prototyps kann im Übersetzungsschritt verloren gehen oder wird verfälscht.

${ }^{3}$ Vgl. Definition bei HeUfLeR [23] 
Um den Einfluss des Zeichenvorrats auf das Prototyping zu erklären, bietet sich die Adapted Media Richness Theory nach SCHMIDT ET AL. [24] an, die den Informationsgehalt eines Prototyps in Relation zu dessen beabsichtigter Aufgabe setzt. Im Falle eines zu geringen Informationsgehalts des Prototyps, kann dieser für den Nutzer als Bewerter mehrdeutig oder missverständlich sein. Analog kann der Kunde im Falle eines zu komplexen Prototypen die wesentlichen Informationen nicht herausfiltern. Beides führt zu nicht belastbarem Feedback (ebd.). Dies deckt sich mit der Anatomie von Prototypen nach Lim et al. [25] insofern, als dass es sich bei Prototypen um filtrierende Kommunikationswerkzeuge handelt. Entsprechend präsentieren Schmidt et al. [24] ein Kommunikationsmodell, dass den Informationsfluss samt entsprechender Filter beschreibt.

Das anschließende Verstehen und „Sich-zu-eigen-machen“ des vorliegenden Prototyps durch den Nutzer im jeweiligen Nutzungskontext kann mit Hilfe von RICœEURS Erweiterung der Hermeneutik auf objektivierbare menschliche Aktionen [26] erklärt werden und deckt sich mit der notwendigen Übereinstimmung der Zeichen im Shannon-Weaver-Modell: Durch Unterschiede in der Interpretation bestehender oder neuer Zeichen geht der Sinn des Prototyps im Übersetzungsschritt verloren oder wird verfälscht.

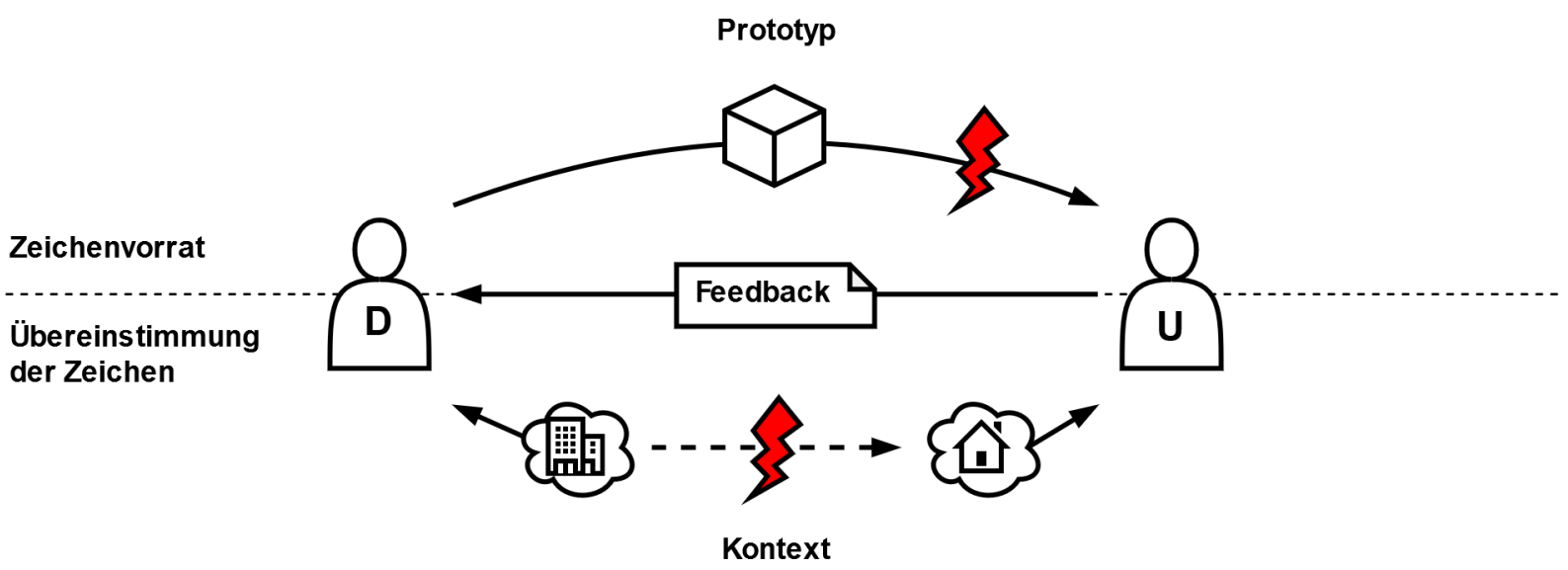

Bild 2: Störeinflüsse zwischen Entwickler (D) und Nutzer (U) im Prototyping als kybernetischer Handlungskreis

Ein Ansatz, um die Position und den Einfluss der Filter zu ändern, wird durch Co-Design beschrieben. Nach SANDERS \& STAPPERS [27], handelt es sich hierbei um eine Form von CoCreation, also der kollektiven Kreativität und Zusammenarbeit während des Entwicklungsprozesses, zwischen Entwicklern und Nutzern. Im Gegensatz zu seiner „klassischen“, passiven Rolle kann der Nutzer hier als "Experte seiner Erfahrungen" [28] am Entwicklungsprozess teilnehmen und in diesem Sinne zum "Entwickler" werden, während der professionelle Entwickler als Unterstützer fungiert [27]. Hierdurch verschieben sich Position und Art der Störgrößen, wie in Bild 3: Der Prototyp wird nun in gemeinsamer Arbeit erstellt. Dies birgt zwar zum einen das Risiko, dass die Voreingenommenheit des Entwicklers das Ergebnis in einer Weise beeinflusst, so dass der Prototyp die ursprünglichen Bedürfnisse des Benutzers nicht korrekt widerspiegelt. Zum anderen erhöht sich jedoch die Anzahl der Modalitäten für den Informationsfluss in Richtung des Entwicklers und schließt die objektbasierten Informationen des Prototyps ein. Zwar erhöht sich damit auch der Interpretations- und Transformationsaufwand, allerdings ist es dem Entwickler möglich, ein tieferes Verständnis für den Nutzerkontext zu entwickeln, sodass die Übereinstimmung der verwendeten Zeichen zunimmt. 


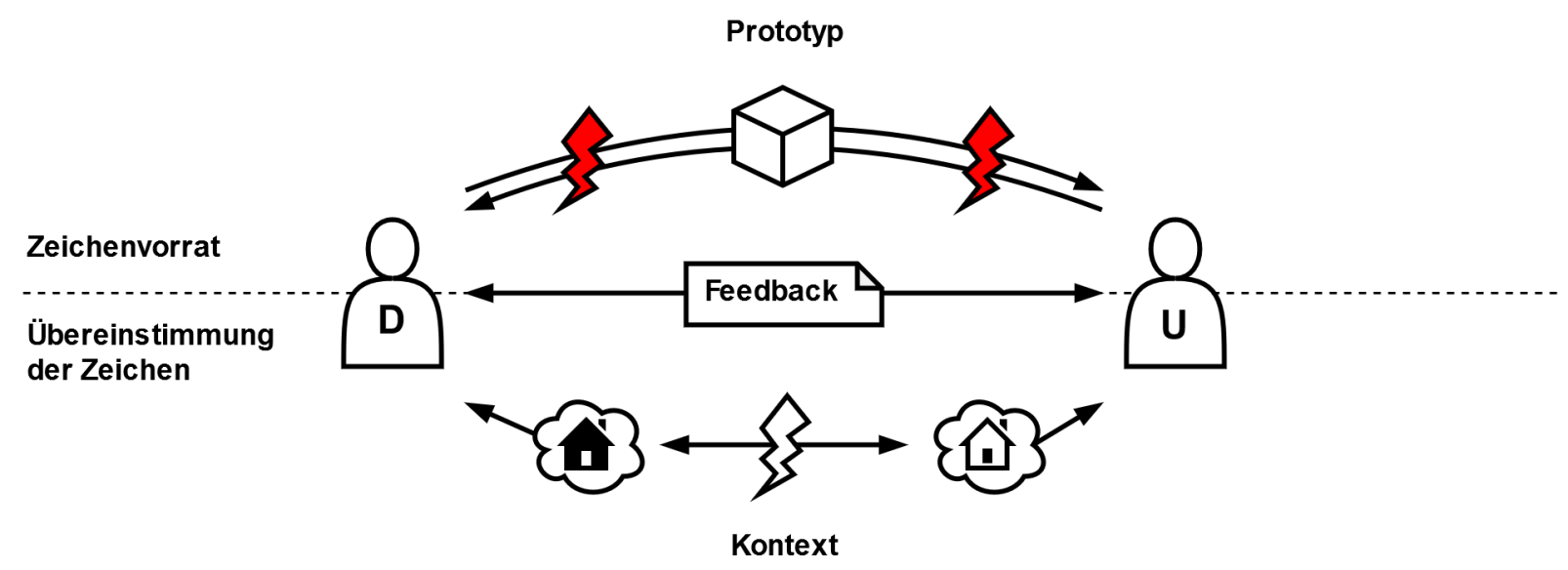

Bild 3: Verschiebung der Störeinflüsse in der Kommunikation zwischen Entwickler (D) und Nutzer (U) im CoDesign.

Denkt man diese Verschiebung zwischen Entwickler und Nutzer weiter, so landet man beim User-Driven Minimum Feasible Product (UD-MFP). Das UD-MFP beschreibt die Artefakte, die von Endnutzern mit ihren begrenzten Ressourcen zur Befriedigung dringender Bedürfnisse geschaffen werden, wenn geeignete Lösungen entweder nicht verfügbar oder unerreichbar sind [29]. Der Nutzer initiiert die Entstehung des UD-MFP (vgl. Bild 4) und konzentriert sich auf die Bedürfnisse, die dringend genug sind, um inn zur Erstellung einer Ad-hoc-Lösung zu veranlassen. Betrachtet man den Entstehungsprozess des UD-MFP, so wird deutlich, dass es zwischen dem Benutzer und dem entstehenden Artefakt keine unterbrechenden Informationsfilter gibt. Die einzigen - aber nichtsdestotrotz wichtigen - Einschränkungen sind die Ressourcen des Benutzers und die hermeneutische Distanz, die sich aus der Manifestation der ursprünglichen Idee ergibt. Das Artefakt stellt also eine originäre Lösung des Problems dar. Aus der Sicht des Entwicklers entsteht jedoch ein Artefakt, das von inm zunächst noch interpretiert werden muss, das heißt, es entsteht zunächst eine erste Problemlösung mit Verbesserungspotenzial in späteren Iterationen.

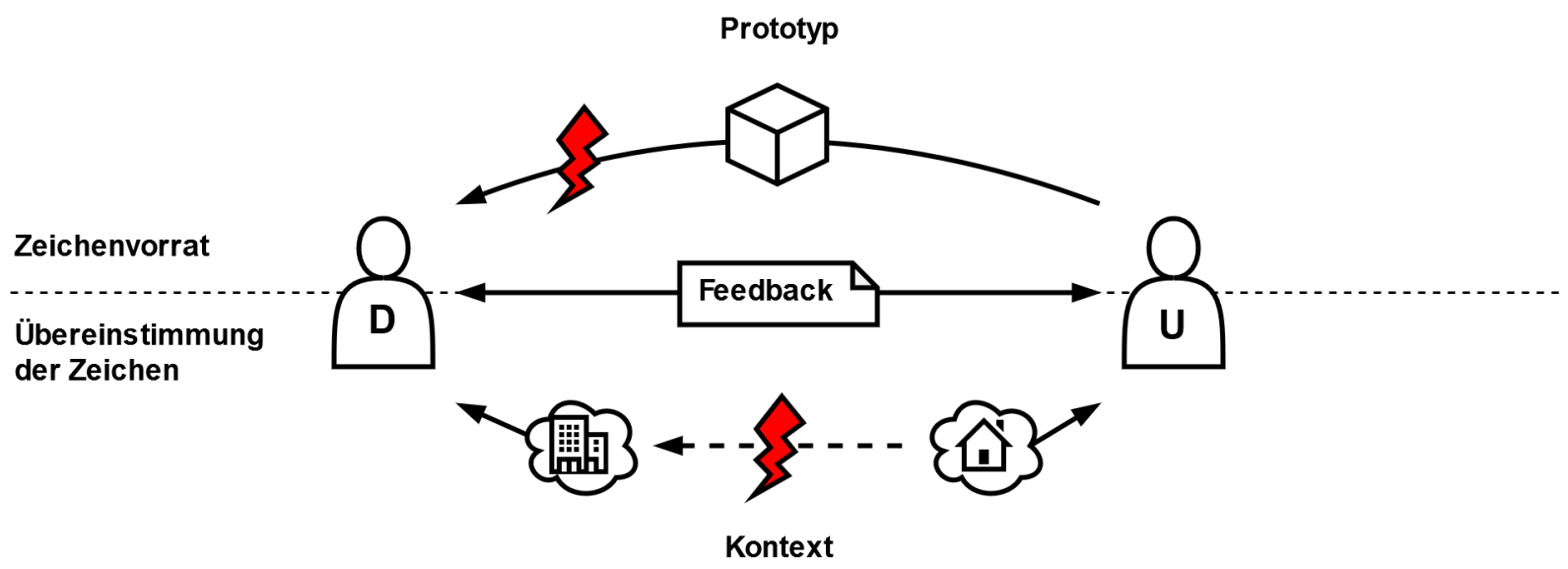

Bild 4: Verschiebung der Störeinflüsse in der Kommunikation zwischen Entwickler (D) und Nutzer (U) im Kontext des UD-MFP.

\section{Diskussion}

Es zeigt sich, dass jegliche Kommunikation zwischen Entwickler und Nutzer gewissen Störgrößen unterliegt. Je nachdem, wie der Benutzer in den Prozess integriert wird, variieren 
jedoch Art und Lage der Störgrößen, und somit auch deren Auswirkung. Dabei können insbesondere zwei verschiedene Arten von Störgrößen identifiziert werden: Zum einen sind dies filtrierende Störungen im Sinne der Media Richness Theory, die in der Art und Darstellung des Prototyps an sich begründet sind. Beispiele für diese Filter können den Arbeiten von SCHMIDT ET AL. [24] und LIM ET AL. [25] entnommen werden. Zum anderen handelt es sich um hermeneutische Verständnislücken, die ihren Ursprung in den unterschiedlichen (Lebens-) Erfahrungen und Kontexten der beteiligten Parteien haben. Hinweise auf deren Einfluss finden sich bei ECKERT ET AL. [5], JAHNKE [30], [31], MøLLER \& TOLLESTRUP [32] und ELVERUM ET AL. [33]. Die Verantwortung für den sorgsamen Umgang mit diesen Abweichungen liegt hier beim Entwickler, der diese im Produktentwicklungsprozess entsprechend berücksichtigen muss, um Effizienz und Validität des gewählten Ansatzes zu gewährleisten.

Insofern stellt sich die Frage nach der Integration in den Entwicklungsprozess: Wie kann der Entwickler den Transformationsprozess besser verstehen und durch gezielte Hilfestellungen im Entwicklungsprozess berücksichtigen?

Dabei kann der Prozess aus zwei verschiedenen Blickwinkeln betrachtet werden: Aus Sicht des Entwicklers und des Nutzers stellen sich die Störgrößen jeweils unterschiedlich dar. Der Entwickler muss daher die Perspektive des Nutzers verstehen: Welche Zeichen kennt der Nutzer? Wie werden diese vom Nutzer interpretiert? Die Antwort auf diese Fragen beeinflusst, mit welchen Gestaltungsmerkmalen ein bestimmter Informationsgehalt transportiert werden kann, um die eingangs avisierte Validierung gezielt durchführen zu können.

Um die Validität der vorliegenden, theoretischen Untersuchung sicherzustellen, folgt sie den Empfehlungen zur theoretischen, strukturellen Validität nach PEDERSEN ET AL. [34] und stützt sich im Verlauf der Argumentation auf bekannte und oft zitierte Theorien der Kommunikation [11], [12], Kybernetik [22] und Hermeneutik [26]. Seit der Veröffentlichung dieser grundlegenden Arbeiten ist keine Falsifikation durch den wissenschaftlichen Diskurs bekannt geworden. Unser Beitrag versteht sich als einzelner, aber grundlegender Schritt hin zur besseren Beherrschbarkeit von Nutzerintegration mit Hilfe von Prototyping. Weitere Untersuchungen sind notwendig - und werden folgen - um die theoretischen Überlegungen in praktisches Wissen zu überführen. Nichtsdestotrotz wollen wir unsere aktuelle Arbeit der wissenschaftlichen Fachgemeinde im Hinblick auf eine fruchtbare Debatte vorstellen.

\section{Zusammenfassung und Ausblick}

Durch die induzierten Abweichungen in der Interpretation der Prototypen entstehen, wie gezeigt, zusätzliche Unsicherheiten im Entwicklungsprozess, die bisher nur mangelhaft erfasst wurden. Um Prototyping in kurzen Iterationszyklen effektiver einsetzen und nutzbar machen zu können, ist daher eine genaue Systembeschreibung des Prozesses samt vorhandener Störgrößen notwendig - je genauer ein System beschrieben werden kann, umso besser lässt es sich beherrschen. Im vorliegenden Beitrag wurden zwei grundlegende Ursachen und Mechanismen für Störungen in der Kommunikation beim Einsatz von Prototypen identifiziert.

Darauf aufbauend kann nun eine Beschreibung der zu erwartenden Auswirkungen bei Eintreten der beschriebenen Störungen folgen: Durch eine qualitative und quantitative Beschreibung der Einflussfaktoren im Prototyping kann der Prozess transparenter für den Anwender gemacht werden. Ziel soll es sein, einen ein Handlungsleitfaden bereitzustellen, der es ermöglicht, Fehlinterpretationen bereits a priori zu vermeiden und damit die Anzahl notwendiger Iterationen im Produktentwicklungsprozess zu reduzieren. 


\section{Literaturverzeichnis}

[1] KRAuse, Dieter ; Gebhardt, Nicolas: Auswirkungen auf die Produktentwicklungsprozesse und zukünftige Trends. In: KRAUSE, D. ; GeBHARDT, N.: Methodische Entwicklung modularer Produktfamilien. Berlin, Heidelberg : Springer Berlin Heidelberg, 2018 - ISBN 978-3-662-53039-9, S. 243-263

[2] LINK, PATRICK: Agile Methoden im Produkt-Lifecycle-Prozess - Mit agilen Methoden die Komplexität im Innovationsprozess handhaben. In: ScHOENEBERG, K.-P. (Hrsg.): Komplexitätsmanagement in Unternehmen. Wiesbaden : Springer Fachmedien Wiesbaden, 2014 — ISBN 978-3-658-01283-0, S. 6592

[3] Buxton, Bill: Sketching User Experiences: Getting the Design Right and the Right Design, Interactive Technologies : Morgan Kaufmann Publishers, 2007 — ISBN 978-0-12-374037-3

[4] Merten, Klaus: Kommunikation: eine Begriffs- und Prozeßanalyse, Studien zur Sozialwissenschaft. Opladen : Westdt. Verl, 1977 - ISBN 978-3-663-01705-9

[5] ECKert, Claudia; Maier, Anja ; McMahon, McMahon: Communication in design. In: Clarkson, J. ; ECKERT, C. (Hrsg.): Design Process Improvement. London : Springer-Verlag, 2005 — ISBN ISBN 978-184628-061-0, S. 232-261

[6] ReINICKE, TAMARA: Möglichkeiten und Grenzen der Nutzerintegration in der Produktentwicklung: Eine Systematik zur Anpassung von Methoden zur Nutzerintegration. Berlin, Technische Universität Berlin, 2004

[7] Wallisch, Anne ; Briede-Westermeyer, Juan Carlos ; Luzardo-Briceño, Marianela: Fostering UserEmpathy Skills of Engineering Students by Collaborative Teaching. In: International Journal of Engineering Education Bd. 36 (2021). - accepted for publication in June 2020

[8] Steffen, D. ; BürdeK, B. E. ; Fischer, V. ; Gros, J. ; Hochschule für Gestaltung Offendach am Main (Hrsg.): Design als Produktsprache: der „Offenbacher Ansatz“ in Theorie und Praxis, Formtheorie. Frankfurt am Main : Verl. Form, 2000 — ISBN 978-3-931317-34-8

[9] SARODNICK, FLORIAN ; BRAU, HENNING: Methoden der Usability Evaluation: wissenschaftliche Grundlagen und praktische Anwendung. 3., unveränderte Auflage. Bern : Hogrefe, 2016 - ISBN 978-3-456-85597-4

[10] RÖHNER, JessicA ; SCHÜTZ, AstRID: Klassische Kommunikationsmodelle. In: RÖHNER, J. ; SCHÜTZ, A.: Psychologie der Kommunikation. Wiesbaden : Springer Fachmedien Wiesbaden, 2016 - ISBN 978-3658-10023-0, S. 19-38

[11] Shannon, Claude Elwood ; Weaver, Warren: The Mathematical Theory of Communication. Urbana : Univ. of Illinois Press, 1998 - ISBN 978-0-252-72548-7

[12] Watzlawick, Paul ; Bavelas, Janet Beavin ; Jackson, Don D.: Menschliche Kommunikation: Formen, Störungen, Paradoxien, Psychologie-Klassiker. 12., unveränd. Aufl. Bern : Huber, 2011 — ISBN 978-3456-84970-6

[13] BoeR, LAURENS ; DonOVAn, JARED: Provotypes for participatory innovation. In: DIS '12: Proceedings of the Designing Interactive Systems Conference. Newcastle Upon Tyne, UK : ACM Press, 2012 — ISBN 9781-4503-1210-3, S. 388-397

[14] BoeR, LAURENS: Participatory Provocation? In: Proceedings of the Participatory Innovation Conference 2011. Sønderborg, Denmark, 2011, S. 18-23

[15] Savola, Alberto: Pretotype It. Second Pretotype Edition., 2011

[16] RIES, ERIC: The Lean Startup: How Today's Entrepreneurs Use Continuous Innovation to Create Radically Successful Businesses : Crown/Archetype, 2011 — ISBN 978-0-307-88791-7

[17] Moogk, DobriLA Rancic: Minimum Viable Product and the Importance of Experimentation in Technology Startups. In: Technology Innovation Management Review (2012), S. 23-26

[18] Duc, Anh Nguyen ; Abrahamsson, PekKa: Minimum Viable Product or Multiple Facet Product? The Role of MVP in Software Startups. In: SHARP, H. ; HALL, T. (Hrsg.): Agile Processes, in Software Engineering, and Extreme Programming. Cham : Springer International Publishing, 2016 - ISBN 978-3-319-33515-5, S. $118-130$

[19] Schmidt, Tobias ; Shaker Verlag GmbH: Towards a Method for Agile Development in Mechatronics A Lead User-based Analysis on How to Cope with the Constraints of Physicality, 2019

[20] Houde, S. ; HILL, C: What do Prototypes Prototype? In: Handbook of Human-Computer Interaction. 2. Aufl. : Elsevier, 1997 - ISBN 978-0-444-81862-1, S. 367-381

[21] Bürdek, Bernhard E.: Design: Geschichte, Theorie und Praxis der Produktgestaltung. 4., überarb. Aufl. Basel : Birkhäuser, 2015 - ISBN 978-3-0356-0404-7

[22] ROPOHL, GüNTER: Allgemeine Technologie: eine Systemtheorie der Technik. 3., überarbeitete Auflage. Karlsruhe : Universitätsverlag Karlsruhe, 2009 — ISBN 978-3-86644-374-7

[23] HEUfLeR, GeRHARD: Design basics: von der Idee zum Produkt. 5., erweiterte und überarbeitete Auflage. Zürich : Niggli, 2016 — ISBN 978-3-7212-0829-0

[24] Schmidt, Tobias Sebastian ; Wallisch, Anne; Böhmer, Annette Isabel ; Paetzold, Kristin ; Lindemann, UDO: Media richness theory in agile development choosing appropriate kinds of prototypes to obtain reliable feedback. In: . Funchal : IEEE, 2017 - ISBN 978-1-5386-0774-9, S. 521-530

[25] LIM, YOUN-KYUNG ; STOLTERMAN, ERIK; TENENBERG, JOSH: The anatomy of prototypes: Prototypes as filters, prototypes as manifestations of design ideas. In: ACM Transactions on Computer-Human Interaction Bd. 15 (2008), Nr. 2, S. 1-27

[26] RICOEUR, PAUL: Der Text als Modell: hermeneutisches Verstehen. In: BüHL, W. L. (Hrsg.): Verstehende Soziologie: Grundzüge und Entwicklungstendenzen. Elf Aufsätze, Nymphenburger Texte zur 
Wissenschaft. München : Nymphenburger Verlagshandlung, 1972 — ISBN 978-3-485-03209-4, S. 252283

[27] Sanders, Elizabeth B.-N. ; Stappers, Pieter Jan: Co-creation and the new landscapes of design. In: CoDesign Bd. 4 (2008), Nr. 1, S. 5-18

[28] Visser, FroukJe SleeswiJk ; Stappers, Pieter Jan ; Lugt, Remko van der ; Sanders, Elizabeth B.-N.: Contextmapping: experiences from practice. In: CoDesign Bd. 1 (2005), Nr. 2, S. 119-149

[29] Nicklas, Simon Jakob; Atzberger, Alexander ; Briede-Westermeyer, JuAn Carlos ; Paetzold, Kristin: The User-Driven Minimum Feasible Product - Towards a Novel Approach on User Integration. In: Proceedings of the DESIGN 2020 16th International Design Conference. Cavtat, HR, 2020

[30] JAHNKE, MARCUS: Revisiting Design as a Hermeneutic Practice: An Investigation of Paul Ricoeur's Critical Hermeneutics. In: Design Issues Bd. 28 (2012), Nr. 2, S. 30-40

[31] JAHNKE, MARCUS: Meaning in the making: introducing a hermeneutic perspective on the contribution of design practice to innovation. Gothenburg, ArtMonitor: Faculty of Fine, Applied and Performing Arts, University of Gothenburg, 2013

[32] MøLLER, LOUISE ; TOLLESTRUP, CHRISTIAN: Creating Shared Understanding in Product Development Teams. London : Springer London, 2013 — ISBN 978-1-4471-4179-2

[33] Elverum, Christer W. ; Welo, TORgeIR ; Tronvoll, Sigmund: Prototyping in New Product Development: Strategy Considerations. In: Procedia CIRP, 26th CIRP Design Conference. Bd. 50 (2016), S. 117-122

[34] Pedersen, KJartan ; Emblemsvåg, Jan ; Consulting, Considium ; Bailey, Reid ; Allen, Janet K ; Mistree, FARROKH: The „Validation Square" - Validating Design Methods \& Research. In: . Baltimore, Maryland, 2000 